\title{
Spatial priming in ecologically relevant reference frames
}

\author{
Sarah M. Tower-Richardi ${ }^{1}$ - Andrew B. Leber ${ }^{1,2}$ • Julie D. Golomb ${ }^{2}$
}

Published online: 30 October 2015

(C) The Psychonomic Society, Inc. 2015

\begin{abstract}
In recent years, researchers have observed many phenomena demonstrating how the visual system exploits spatial regularities in the environment in order to benefit behavior. In this paper, we question whether spatial priming can be considered one such phenomenon. Spatial priming is defined as a response time facilitation to a visual search target when its spatial position has been repeated in recent trials (Maljkovic \& Nakayama, 1996, Perception \& Psychophysics, 58, 977-991). Does this priming serve a behaviorally adaptive role or is it merely a byproduct of ongoing visual processing? Critically, an adaptive priming mechanism must actively transform visual inputs from native retinotopic (eye-centered) coordinates into ecologically relevant coordinates, e.g., spatiotopic (world-centered) and/or object-centered. In Experiment 1, we tested this hypothesis by having participants move their eyes between trials, which dissociated retinotopic and spatiotopic frames of reference. Results showed only weak retinotopic priming, but robust spatiotopic priming. The second experiment again had participants move their eyes between trials but also manipulated the placement of a grouped array of display objects from trial to trial. This allowed us to measure not just retinotopic and spatiotopic priming, but object-centered priming as well. Results from
\end{abstract}

Andrew B. Leber

leber.30@osu.edu

Julie D. Golomb

golomb.9@osu.edu

1 Department of Psychology, University of New Hampshire, Durham, NH, USA

2 Department of Psychology, The Ohio State University, 225 Psychology Building, 1835 Neil Avenue, Columbus, OH 43210 , USA this experiment did not yield retinotopic priming but showed robust spatiotopic and object-centered priming. These findings demonstrate that spatial priming operates within ecologically relevant coordinate systems, and the findings support the notion that spatial priming serves an adaptive role in human behavior.

Keywords Priming - Attention: space-based - Attention: object-based

Although the world is largely dynamic, much of the information that is available to the human observer remains stable over time. This stability offers a set of spatial regularities that, when exploited by the visual system, can benefit behavior. For example, we are able to navigate our offices more efficiently once we learn the consistent locations of behaviorally relevant objects (e.g., door, light switch, chair, etc.).

An abundance of research in recent years has demonstrated ways in which the visual system learns to exploit spatial regularities. In one example, probability cueing, manipulations place targets - e.g., a T among Ls - in "rich" portions of a visual search display with a greater frequency than in "sparse" portions. Within a few minutes, observers begin to search preferentially for targets in the rich portions of the display (Geng \& Behrmann, 2002; Hoffmann \& Kunde, 1999; Miller, 1988; Jiang, Swallow, Rosenbaum \& Herzig, 2013). In contextual cueing, the spatial arrangements of the search items on "predictive" trials are repeated once per block throughout the experiment. For each of these predictive displays, the target occupies a fixed location, so the spatial arrangement, or context, acts as a cue for the target location. Within a few blocks of trials, observers are faster to find targets on predictive trials than on non-predictive trials (Chun \& Jiang, 1998; Chun, 2000; Jiang \& Wagner, 2004). 
Beyond these two well-studied phenomena, several more reports have corroborated that spatial regularities are learned routinely by observers and used to re-weight the manner in which information is prioritized in the spatial domain (Anderson, 2014; Chelazzi et al., 2014; Fiser \& Aslin, 2001; Lee \& Shomstein, 2013; Shomstein \& Johnson, 2013). From an ecological perspective, these examples all have a clear adaptive purpose: they make behavior more efficient.

In this paper, we consider the ecological validity of another phenomenon, spatial priming. Also called position priming, this phenomenon is defined as a response time (RT) facilitation to a visual search target when its spatial position has been repeated in recent trials (Maljkovic \& Nakayama, 1996; see also Ball, Smith, Ellison, \& Schenk, 2009; Geyer, Müller \& Krummenacher, 2007; Kumada \& Humphreys, 2002; Leber, Lechak \& Tower-Richardi, 2013; Rabbitt, Cumming, \& Vyas, 1977). In a seminal study using pop-out search, Maljkovic and Nakayama (1996) presented observers with trials containing three stimuli, one of which was a color oddball (e.g., a red target among green non-targets). Faster RTs were found when the pop-out target appeared in a location that had previously been occupied by a target. Additionally, a complementary inhibition effect was observed, in which RTs were slowed when the target stimulus appeared in a location that had been previously occupied by a distractor (see also Geyer et al., 2007; 2010). Maljkovic and Nakayama observed these priming effects to be driven most strongly by the trial immediately preceding the current trial (i.e., the $n-1$ trial, where $n$ represents the current trial), but the effects were also reliably driven by stimuli from as early as the $n-8$ trial.

From an ecological perspective, spatial priming raises an interesting question because it offers no net behavioral advantage, at least in the laboratory; due to the random placement of the target on each trial, the occasional benefit from repeated target locations is offset by the relative disadvantage of switched target locations. Yet participants still demonstrate spatial priming. Why? We consider two possible explanations.

By an adaptive account, the visual system is designed to prioritize recently selected spatial locations because doing so is usually useful. Maljkovic and Nakayama (1996) adopted such a viewpoint, arguing that spatial priming could be beneficial for sequences of actions where attention to specific locations is commonly repeated over the short-term. For example, drinking a cup of coffee typically involves periodically reaching to the same location to both lift and return the cup. Similarly, when a motorist is waiting at an intersection, at a red light, she will need to repeatedly attend to the traffic light's stationary location until the light turns green. Thus, priming could help reinforce brief episodes of attentional engagement to objects of interest in the real world.

In contrast, according to a byproduct account, priming might not exist to benefit performance but is instead an architectural byproduct of other adaptive visual processes. One example is the motion aftereffect, which does not in any way optimize behavior but rather results from how motion selective neurons adapt to prolonged stimulation (e.g., Anstis, Verstraten \& Mather, 1998). In the case of spatial priming, it could simply be a residual persistence of spatial priority from trial to trial.

One way to tease these two accounts apart is to ask what reference frame spatial priming operates in. The adaptive account would argue that priming should occur in behaviorally relevant coordinates and should be robust to events such as eye movements. Our visual system receives information in eye-centered (retinotopic) coordinates, and this information changes constantly as our eyes scan the visual world. It is rarely beneficial to maintain objects of interest in these everchanging retinotopic coordinates; the most behavioral relevance should be accorded from facilitating stable worldcentered (spatiotopic) or object-centered locations. Thus, an adaptive account would predict spatial priming to occur in one of these more relevant coordinates. The byproduct account, on the other hand, would predict that priming should persist in native (i.e., retinotopic; Golomb, Chun \& Mazer, 2008) coordinates.

Spatial priming can be discussed within the framework of a priority map (also commonly referred to as a salience map or activation map), which represents all locations in the visual field and assigns each of them an activation weight, based on stimulus-driven salience and goal-driven factors. During visual search, attentional selection is granted to the location on the priority map with the highest activation level (e.g., Bisley \& Goldberg, 2010; Schall, 1995; Serences \& Yantis, 2006; Wolfe, 1994). Spatial priming can be expressed as a carryforward of the priority map from one trial to the next. That is, the starting activation weights on a given trial are inherited from the final activation weights at the moment of target selection on the previous trial. Given that the target location gains the highest activation level on one trial, the persistence of that activation into the subsequent trial explains the facilitated performance to repeated targets. ${ }^{1}$

Attentional priority maps are thought to be organized in native retinotopic (eye-centered) coordinates. In a series of behavioral and neuroimaging studies, Golomb and colleagues demonstrated that, even when subjects are explicitly instructed to attend to a spatiotopic (world-centered) location, attentional facilitation lingers at the previously relevant retinotopic location for a brief period of time after each eye movement (Golomb et al., 2008; Golomb, Marino, Chun \&

\footnotetext{
${ }^{1}$ While we invoke the priority map, spatial priming could manifest at a later stage of processing, after attentional selection (e.g., episodic retrieval). Such a conception is central to theories of feature-based priming (e.g., Huang, Holcombe \& Pashler, 2004; Hillstrom, 2000; but see Becker, 2008; Yashar \& Lamy, 2010; for a review, see Kristjánsson \& Campana, 2010), although the processing stage of spatial priming has been less explored.
} 
Mazer, 2011; Golomb, L'Heureux, \& Kanwisher, 2014; Golomb, Nguyen-Phuc, Mazer, McCarthy, \& Chun, 2010; Golomb, Pulido, Albrecht, Chun, \& Mazer, 2010). After 100-200 ms, this "retinotopic attentional trace" decays, and attention is able to fully update to the correct spatiotopic location. Critically, the priority maps only appear to update to spatiotopic coordinates when doing so is behaviorally relevant-if the task is to maintain attention on a retinotopic location, attention never updates to the spatiotopic location (Golomb et al., 2008).

Thus, if spatial priming is adaptive and confers a behavioral benefit, then we should expect the underlying priority maps to update to behaviorally relevant coordinates, and we should see the effects of priming in spatiotopic and/or object-centered coordinates after an eye movement (assuming we wait long enough after the eye movement for the updating to complete). On the other hand, if priming is simply a persistent byproduct of recent activation in the existing priority maps, then it should only remain in native retinotopic coordinates after an eye movement.

In the present study, we systematically explore spatial priming effects in multiple reference frames. Our approach was to adapt Maljkovic and Nakayama's (1996) study but with the inclusion of eye movements between trials, which allows a clear dissociation of priming in retinotopic coordinates versus other references frames.

We should note that our study is not the first to examine spatial priming across multiple reference frames. Maljkovic and Nakayama (1996) addressed it themselves in their third experiment. They arranged one target and two distractors in a horizontal array that could be presented in any of the four display quadrants on each trial, while eye gaze was held at a central fixation point. They then presented trials in sequences of as many as nine trials in a row, in which the relative position of the target within the array (i.e., left, center, or right) was held constant but the absolute position of the array (i.e., one of the four display quadrants) varied. They found an RT benefit for the repetitions of relative position, even when the array moved. These results suggested that the spatial priming is not tied to retinotopic coordinates. However, one key limitation of this experiment was that the relative position of the target was predictable due to the repeated trial sequences, and participants may have explicitly picked up on these probabilities. Thus these studies may have included an explicit, goal-driven strategy effect in addition to the pure priming effect, since it would have been advantageous for observers to deliberately attend to the within-array position. Indeed, target predictability has been shown to yield RT benefits above and beyond standard spatial priming (Geyer \& Müller, 2009). This limitation also applies to subsequent studies that examined spatial priming across multiple reference frames (e.g., Ball, Smith, Ellison \&
Schenk, 2009; 2010; Ball, Lane, Ellison \& Schenk, 2011 ), in that it was not possible to disentangle the potential contributions of spatial priming from strategic goal-driven attention based on explicit knowledge. Given the broad array of goal-driven attentional phenomena documented in the literature, this distinction is criti$\mathrm{cal}$, and we thus removed potential goal-driven components from our experimental design.

In the current experiments, we made the target location unpredictable on each trial, ensuring that the trial sequences did not encourage participants to form spatial expectancies and that repetition effects could only be the result of spatial priming. In Experiment 1, we asked whether priming persists after an eye movement, and if so, whether it persists in retinotopic and/or spatiotopic coordinates. We found robust spatiotopic priming and only modest evidence for retinotopic priming. Because there are multiple non-retinotopic reference frames that could have contributed to this "spatiotopic" effect, including world-centered, object-centered, head-centered, body-centered, etc., in Experiment 2 we attempted to further dissociate these reference frames by incorporating both eye movements and "object" movements between trials. To do so, we analyzed target repetitions within a grouped array that could move from trial to trial, allowing for the independent analysis of retinotopic, spatiotopic, and object-centered priming. We found robust spatiotopic and object-centered priming, with negligible retinotopic priming. We argue that these results largely support the adaptive account of spatial priming.

\section{Experiment 1A and 1B}

To first disentangle retinotopic from spatiotopic priming, we designed a task in which the fixation location was chosen randomly between two positions on each trial. This meant that half of the trials involved an eye movement between trials, while for the other half fixation remained in the same location as the previous trial. We aimed to ensure that our findings would be generalizable across both vertical and horizontal eye movements, so we asked participants to move their eyes horizontally in Experiment $1 \mathrm{~A}$ and vertically in Experiment 1B. If priming occurs in retinotopic coordinates, then, following the eye movement, the target in the same retinotopic position (i.e., same position relative to the eyes) will show a facilitated RT compared to a control location. However, if priming occurs in spatiotopic coordinates, then, following the eye movement, the target in the same spatiotopic position (i.e., same location in the display) will show a facilitated RT. Note that these alternatives are not mutually exclusive. However, if we find any spatiotopic effect, it will support the notion that spatial priming operates in ecologically relevant coordinates. 


\section{Method: Experiment 1A and 1B}

\section{Participants}

Sixteen participants ( 11 female; mean age $=26.81$ years $)$ took part in Experiment 1A, and 18 participants (9 female; mean age $=26.22$ years) in Experiment 1B. All participants had selfreported normal or corrected-to-normal visual acuity and color vision. Informed consent was obtained for all participants, and the study protocols were approved by the Massachusetts Institute of Technology Committee On the Use of Humans as Experimental Subjects and the Institutional Review Board of the University of New Hampshire. All participants were compensated US $\$ 10 / \mathrm{h}$. The experiment lasted no more than $1.5 \mathrm{~h}$.

\section{Experimental setup}

Stimuli were generated using MATLAB (Mathworks, Natick, MA) with the Psychtoolbox extensions (Brainard, 1997; Pelli, 1997) and presented on a 22-inch flatscreen CRT monitor with a $4: 3$ aspect ratio. Participants were seated at a chinrest $63.5 \mathrm{~cm}$ away from the monitor. From this distance, the display subtended $35.0^{\circ}$ by $28.0^{\circ}$ visual angle. Eye position was monitored using an eye-tracking system (ISCAN ETL 400 eye tracker) recording pupil and corneal reflection position at $240 \mathrm{~Hz}$.

The eye-tracker was calibrated before beginning the experiment and repeated throughout the experiment as needed. All participants completed a practice phase, which lasted about 2$4 \mathrm{~min}$, before beginning the test phase of the experiment. Data from the practice phase were not analyzed.

\section{Stimuli}

Search displays, which consisted of six stimuli, were generated by drawing a diamond that was $1.91^{\circ}$ in height and width and then truncating its left or right side by $0.68^{\circ}$ (see Fig. 1). In Experiment 1A (horizontal arrangement), stimuli were arranged in two rows of three. The top and bottom rows were centered $4.37^{\circ}$ above and below the center of the screen, respectively, yielding a distance of $8.74^{\circ}$ between rows. In each of the rows, the middle item was centered on the screen's vertical midline, accompanied by one item that was centered $8.74^{\circ}$ to the left and another centered $8.74^{\circ}$ to the right of the middle item. In Experiment 1B (vertical arrangement), the display was oriented vertically, resulting in two columns of three. The locations of these items were determined by simply rotating the Experiment $1 \mathrm{~A}$ display by $90^{\circ}$ about the center of the screen.

A white plus sign (RGB: 255, 255, 255) was used as the fixation cross $\left(0.55^{\circ} \times 0.55^{\circ}\right.$; stroke $\left.=0.05^{\circ}\right)$. In Experiment $1 \mathrm{~A}$, it was centered either $4.37^{\circ}$ to the left or $4.37^{\circ}$ to the right of the center of the screen, such that it was always equidistant from the four items that it was adjacent to. In Experiment 1B, the fixation cross was centered either $4.37^{\circ}$ above or below the center of the screen. The stimuli were presented on a black background (RGB: 0, 0, 0).

Each display consisted of one uniquely colored target stimulus and five distractors of a different color. The color of the target diamond in each display was sampled randomly with replacement from a set of three possible colors: red (RGB: 230, 0, 0), green (RGB: 0, 160, 0), or blue (RGB: 0, 0, 255). The five distractor diamonds were uniform in color and sampled randomly with replacement from the two remaining nontarget colors (e.g., if the target was red, the five distractors would all be either green or blue). The truncated side of each diamond was independently sampled randomly with replacement on each trial.

\section{Procedure}

Each trial began with a 1000-ms presentation of the fixation cross, after which the search display appeared. The task was to stay fixated on the cross and identify which side of the uniquely colored target diamond was truncated. Participants responded by pressing either the ' 1 ' or ' 2 ' on the keyboard number-pad if the left or the right side of the diamond was truncated, respectively. The stimuli remained on the screen for up to $3000 \mathrm{~ms}$ or until participants responded, whichever came first. Participants were instructed to respond quickly while keeping mistakes to a minimum. Both accuracy and RT were recorded.

For trials containing either a response or fixation error, a 1000-ms feedback message was presented at the center of the screen after the trial. For incorrect response or no response trials, "response error" was displayed; for fixation errors, "fixation error" was displayed. A fixation error was made if the participant's eye position deviated more than $2^{\circ}$ in any direction from the correct fixation location. Trials were not aborted if fixation was broken, but these trials were excluded from analyses (see below). No feedback was provided for correct trials. A blank intertrial interval of $500 \mathrm{~ms}$ preceded the start of the next trial.

Each participant performed the task for $1.5 \mathrm{~h}$, or until they completed eight 144-trial blocks, whichever came first. Participants received brief breaks twice during each block.

\section{Design}

There were 12 basic target configurations: two fixation locations crossed with six target locations. Each configuration was presented 12 times per block (in randomized order) to create blocks of 144 trials.

We presented each of the 12 configurations with equal frequency to ensure proper counterbalancing of the 

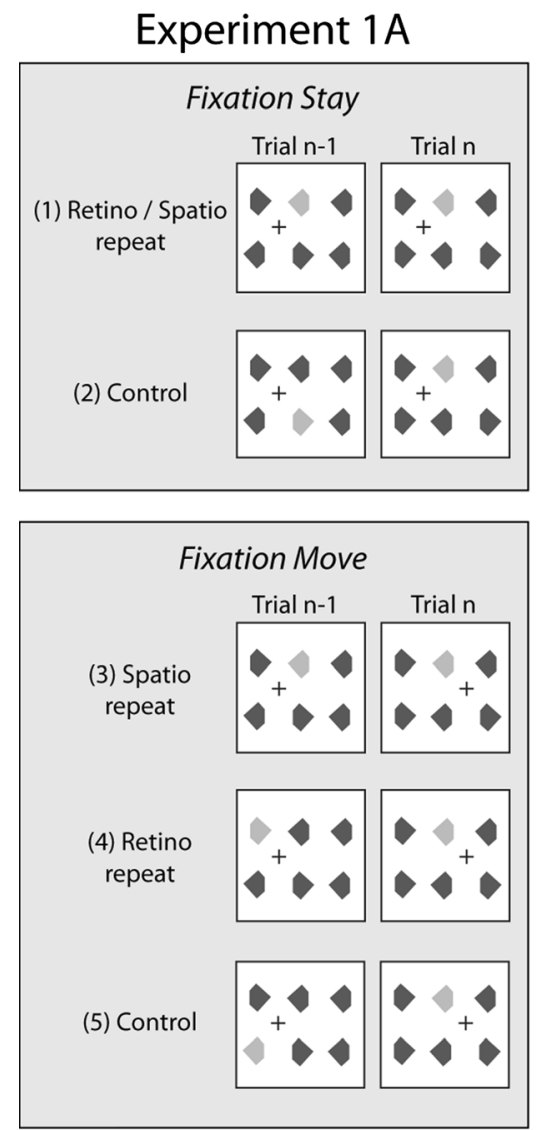

Fig. 1 Representation of stimulus conditions used in Experiments 1A (horizontal arrangement) and 1B (vertical arrangement), shown on left and right sides, respectively. For each respective experiment, sample stimuli of the previous $(n-1)$ and current $(n)$ trials from five conditions are shown. Top panels, trials in which the fixation stays in the same location on both the $n-1$ and $n$ trial; bottom panels trials in which the

stimulus characteristics as well as participant expectations of random variation. However, because our aim in this study was to examine effects of priming, which are determined by similarities in stimuli across successive trials, our analysis needed to focus on a pre-defined set of intertrial conditions (Fig. 1):

(1) fixation stay/all repeat (fixation location and target locations are the same as in the $n-1$ trial; both retinotopic and spatiotopic locations repeat)

(2) fixation stay/control (fixation location is the same as in the $n-1$ trial, target location is different)

(3) fixation move/spatio repeat (fixation location is different from $n-1$ trial, target appears in the same spatiotopic location as in the $n-1$ trial)

(4) fixation move/retino repeat (fixation location is different from the $n-1$ trial, target appears in the same retinotopic location as in the $n-1$ trial)

(5) fixation move/control (fixation location is different from $n-1$ trial, target appears in a location that is
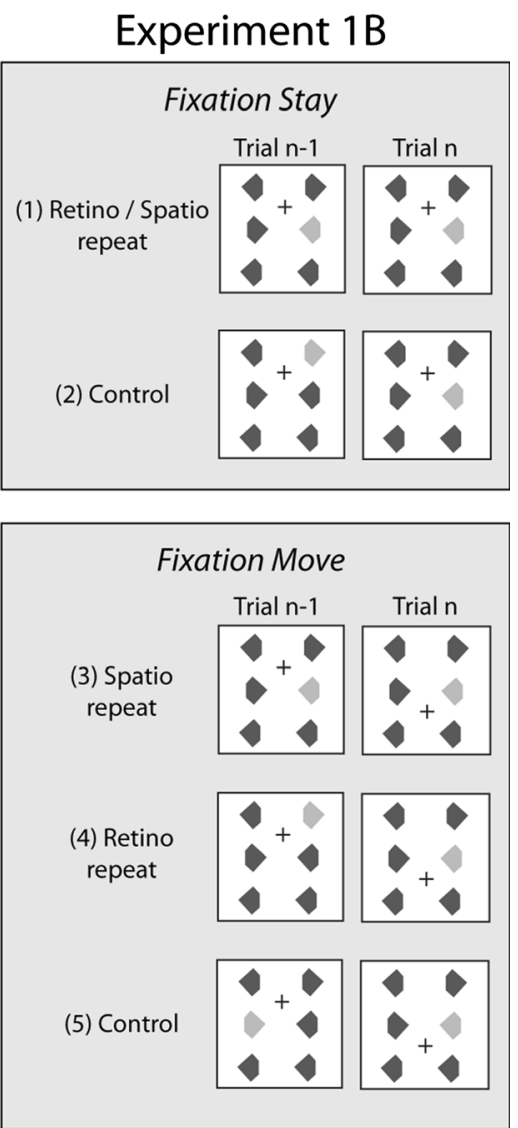

fixation moves between the $n-1$ and $n$ trial. Targets are shown here as the single bright object and distractors as the darker objects, although stimuli were chosen from one of three colors in the actual experiment. Note that each of these rows depicts just one sample configuration out of many possible per condition; see Method for additional details

neither the same spatiotopic nor retinotopic location as in the $n-1$ trial).

Because we ensured that each target configuration was presented equally often, the five intertrial conditions did not occur with equal frequency. The mean expected number of trials per block (predicted by chance) for each of the intertrial conditions is shown in Table 1; expected trial counts are greater in some intertrial conditions than others. However, these expected counts are only probabilities; the actual frequencies of the intertrial conditions are dependent upon the specific presentation order of the trials in each block. To ensure that chance deviations from the expected trial counts in individual blocks did not yield too few observations for any of the intertrial conditions (especially the fixation stay/all repeat, fixation move/spatio repeat, and fixation move/retino repeat conditions, which yielded low expected frequencies), we performed a randomization procedure prior to each block that iteratively generated and tested multiple possible trial sequences until finding a sequence that conformed to the following 
Table 1 Condition characteristics and results from Experiment 1A and 1B. Retino retinotopic, spatio spatiotopic, $R T$ response time, $m s$ milliseconds

\begin{tabular}{|c|c|c|c|c|c|c|c|c|c|c|}
\hline \multirow[t]{2}{*}{ Condition } & \multirow{2}{*}{$\begin{array}{l}\text { Trials/block predicted by } \\
\text { chance }\end{array}$} & \multirow{2}{*}{$\begin{array}{l}\text { Actual range of } \\
\text { trials/block }\end{array}$} & \multicolumn{4}{|c|}{ Experiment $1 \mathrm{~A}$} & \multicolumn{4}{|c|}{ Experiment 1B } \\
\hline & & & $\begin{array}{l}\mathrm{RT} \\
(\mathrm{ms})\end{array}$ & $\begin{array}{l}\text { Error } \\
(\%)\end{array}$ & $\begin{array}{l}\text { RT } \\
\text { priming } \\
(\mathrm{ms})^{\mathrm{a}}\end{array}$ & $\begin{array}{l}\text { Accuracy } \\
\text { priming } \\
(\%)^{\mathrm{a}}\end{array}$ & $\begin{array}{l}\mathrm{RT} \\
(\mathrm{ms})\end{array}$ & $\begin{array}{l}\text { Error } \\
(\%)\end{array}$ & $\begin{array}{l}\text { RT } \\
\text { priming } \\
(\mathrm{ms})^{\mathrm{a}}\end{array}$ & $\begin{array}{l}\text { Accuracy } \\
\text { priming } \\
(\%)^{\mathrm{a}}\end{array}$ \\
\hline \multicolumn{11}{|l|}{ Fixation stay } \\
\hline $\begin{array}{l}\text { (1) Retino/spatio } \\
\text { repeat }\end{array}$ & 12 & $12-14$ & 600 & 2.05 & $40^{*}$ & 1.32 & 612 & 1.72 & $31^{*}$ & 1.74 \\
\hline (2) Control & 60 & Not constrained & 640 & 3.37 & - & - & 643 & 3.46 & - & - \\
\hline \multicolumn{11}{|l|}{ Fixation move } \\
\hline (3) Spatio repeat & 12 & $12-14$ & 604 & 2.16 & $32 *$ & 0.41 & 621 & 1.55 & $27 *$ & 0.52 \\
\hline (4) Retino repeat & 8 & $8-10$ & 634 & 2.03 & 2 & 0.54 & 635 & 0.98 & $31 *$ & 1.09 \\
\hline (5) Control & 52 & Not constrained & 636 & 2.57 & - & - & 648 & 2.07 & - & - \\
\hline
\end{tabular}

* Significant at alpha $=0.05$ after Holm-Bonferroni correction for multiple comparisons

${ }^{a}$ Priming was defined as the value for each repeat condition subtracted from its corresponding control condition (see Experiment 1A and 1B Results for details), and thus priming measures were not applicable to control conditions

constraints: Exactly $50 \%$ of trials were fixation stay and $50 \%$ were fixation move, and no more than five trials in a row contained the same fixation location. Additionally, we constrained the randomization to yield a specific range of trial counts per block for each of the low-frequency conditions; these ranges are reported as "Actual range of trials/block" in Table 1. This procedure ensured sufficient statistical power for each intertrial condition while remaining close to chance level.

To ensure sufficient statistical power in our analyses, participants who completed fewer than 20 trials (after removing fixation errors and response errors) in any of the five intertrial conditions were excluded from analysis. These criteria led us to exclude one participant in Experiment $1 \mathrm{~A}$ and two participants in Experiment 1B.

\section{Experiment $1 \mathrm{~A}$}

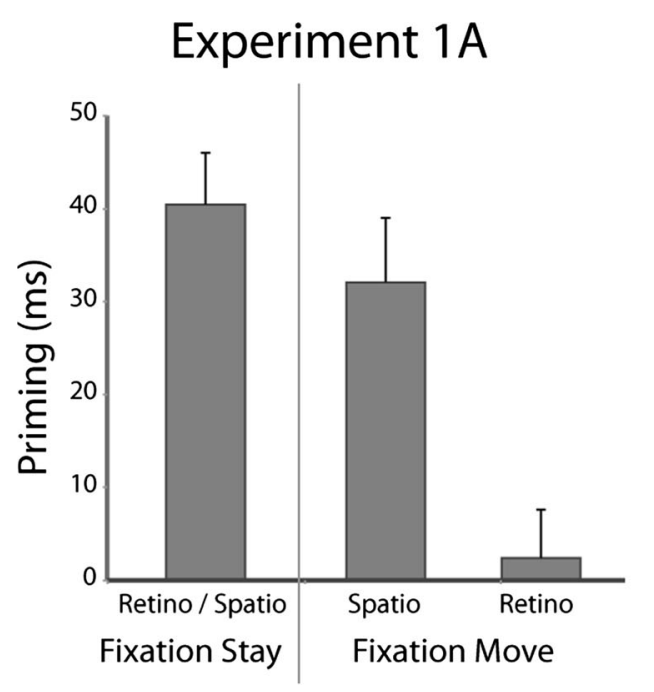

\section{Results: Experiment 1A (horizontal arrangement)}

\section{Overall performance}

Mean fixation accuracy was $88.87 \%(\mathrm{SD}=8.99 \%)$; all trials with fixation errors were excluded from further analysis. Overall response accuracy was $97.03 \%(\mathrm{SD}=3.36 \%)$. RT data were trimmed at $3 \mathrm{SD}$ above each participant's mean, separately for trials with targets that were close and far in eccentricity (i.e., adjacent to fixation and non-adjacent, respectively). Overall, trimmed RTs were faster for closeeccentricity trials than far-eccentricity trials, $638 \mathrm{~ms}$ and $699 \mathrm{~ms}$, respectively, $t(14)=13.01, P<0.001, d=3.359$. Because we did not explicitly match eccentricity across

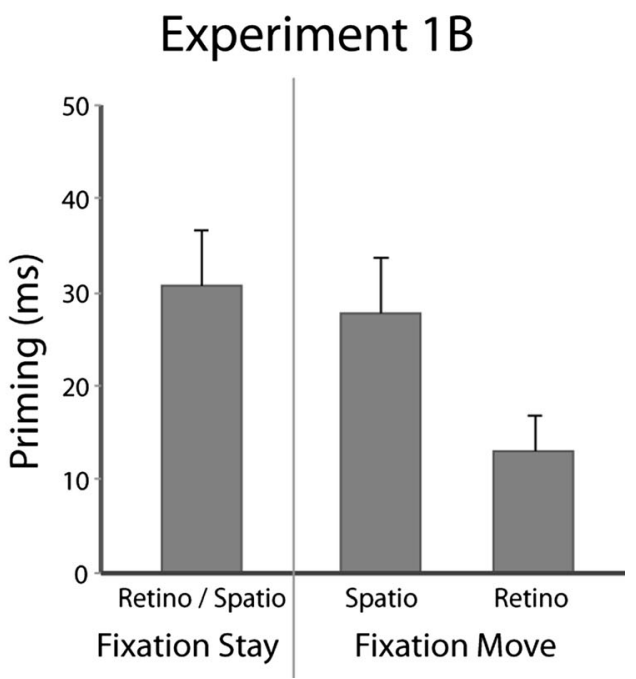

Fig. 2 Results from Experiment 1A and 1B, shown on left and right sides, respectively. Priming is computed as reaction time (RT) differences between the labeled condition and its corresponding control (see Fig, 1 or Table 1). Error bars: Standard error of the mean (SEM) 
intertrial conditions, it was important to remove this potentially confounding factor by separately analyzing trials with close and far eccentricities. Since we did not have enough statistical power to separately examine the far trials (which were substantially less frequent than close trials), we restricted the remaining analyses to trials in which the target was close in eccentricity on both the present and previous $(n-1)$ trial.

\section{Spatial priming effects}

Reaction time Mean RTs for each of the five intertrial conditions are reported in Table 1. The "repeat" conditions (fixation stay/all repeat, fixation move/spatio repeat, fixation move/ retino repeat) were compared to their respective control conditions (fixation stay/control, fixation move/control), to yield three distinct measures of spatial priming (shown in Fig. 2 and Table 1). We tested each of these for statistical significance, using paired-samples $t$-tests with a Holm-Bonferroni correction for familywise error (Holm, 1979):

(1) Retino/Spatio Priming (Fixation Stay). When fixation position was held constant we found significant priming, $t(14)=7.26, P_{\text {adjusted }}=0.00001, d=1.87$, replicating the classic spatial priming result (e.g., Maljkovic \& Nakayama, 1996, Experiment 1). On these fixation stay trials, retinotopic and spatiotopic contributions cannot be disentangled; thus, priming in this case can be thought of as retinotopic and/or spatiotopic.

(2) Spatio Priming (Fixation Move). Significant priming was found in spatiotopic coordinates after an eye movement, when the target repeated the same spatiotopic location compared to control: $t(14)=4.62, P_{\text {adjusted }}=$ $0.00079, d=1.19$.

(3) Retino Priming (Fixation Move). In contrast to (2), we did not find significant retinotopic priming, $t(14)=0.46$, $P_{\text {adjusted }}=0.655, d=0.12$.

To directly compare the three estimates of spatial priming, we entered them into a one-way ANOVA, which reached significance, $F(2,28)=12.894, P=0.0001, \eta_{\mathrm{p}}{ }^{2}=$ .479. Post-hoc comparisons, using the Holm-Bonferroni correction, showed that Retino/Spatio Priming (Fixation Stay) was not significantly different from Spatio Priming (Fixation Move), $t(14)=1.06, P_{\text {adjusted }}=0.308, d=0.27$. However, Retino/Spatio Priming (Fixation Stay) was significantly greater than Retino Priming (Fixation Move), $t(14)=$ 4.677, $P_{\text {adjusted }}=0.001, d=1.21$. Also, Spatio Priming (Fixation Move) was greater than Retino Priming (Fixation Move), $t(14)=3.895, P_{\text {adjusted }}=0.003, d=1.01$.

Accuracy Accuracy was analyzed in a similar fashion to RT. Mean error rates for the five intertrial conditions are reported in Table 1. From these conditions, we computed the three accuracy priming scores, which are also reported in Table 1. Holm-Bonferroni corrected paired-samples T-tests did not reveal significant accuracy priming in any of the three conditions, and a one-way ANOVA did not show any significant differences among the priming scores.

\section{Results: Experiment 1B (vertical arrangement)}

\section{Overall performance}

Mean fixation accuracy was $83.74 \%(\mathrm{SD}=11.05 \%)$, and all trials with fixation errors were excluded from further analysis. Overall response accuracy was $97.66 \%(\mathrm{SD}=1.89 \%)$. RT data were trimmed at $3 \mathrm{SD}$ above each participant's mean, separately for trials that were close and far from fixation. Overall trimmed RTs were faster for close-eccentricity trials than far-eccentricity trials, $644 \mathrm{~ms}$ and $716 \mathrm{~ms}$ respectively, $t(15)=10.70, P<0.0001, d=2.675$. As in Experiment 1A, we restricted the remaining analyses to close trials only.

\section{Spatial priming effects}

Reaction time Mean RTs for each of the five intertrial conditions are reported in Table 1, and spatial priming was analyzed as in Experiment 1A:

(1) Retino/Spatio Priming (Fixation Stay). Results again revealed significant priming in the combined spatiotopic/ retinotopic case, $t(15)=5.00, P_{\text {adjusted }}=0.0005, d=$ 1.25 .

(2) Spatio Priming (Fixation Move). As in Experiment 1A, priming was significant in spatiotopic coordinates, $t(15)$ $=4.58, P_{\text {adjusted }}=0.0007, d=1.15$.

(3) Retino Priming (Fixation Move). Significant priming was also observed in retinotopic coordinates in this experiment, $t(15)=3.46, P_{\text {adjusted }}=0.0035, d=0.87$, unlike Experiment $1 \mathrm{~A}$.

The one-way ANOVA comparing the three priming measures again reached significance, $F(2,30)=4.923$, $P=0.014, \eta_{\mathrm{p}}{ }^{2}=.247$. Holm-Bonferroni corrected post-hoc comparisons showed that Retino/Spatio Priming (Fixation Stay) was not significantly different from Spatio Priming (Fixation Move), $t(15)=0.52, P_{\text {adjusted }}=0.608, d=0.13$. However, Retino/Spatio Priming (Fixation Stay) was significantly greater than Retino Priming (Fixation Move), $t(15)=2.83, P_{\text {adjusted }}=0.038, d=0.71$. Also, Spatio Priming (Fixation Move) was marginally significantly greater than Retino Priming (Fixation Move), $t(15)=2.38$, $P_{\text {adjusted }}=0.062, d=0.60$. 
Accuracy Mean error rates and accuracy priming scores are reported in Table 1. As in Experiment 1A, we did not find any significant priming effects with accuracy measures.

\section{Discussion: Experiment 1A and B}

In Experiment $1 \mathrm{~A}$ and $\mathrm{B}$ we replicated the well-documented spatial priming effect in which faster RTs are found to targets presented in the same location as the previous trial, when fixation location is not varied (Maljkovic \& Nakayama, 1996). Moreover, we found that spatial priming survives an eye movement, and it is observed primarily in spatiotopic coordinates. While we did obtain some evidence for retinotopic priming in Experiment 1B, it was weaker than the spatiotopic effect. Given that the spatiotopic location is more ecologically relevant than the retinotopic location, this suggests that priming respects ecologically relevant coordinates and is not simply an architectural byproduct of the visual system, although there may be some byproduct priming that also exists.

\section{Experiment 2}

The main goal of the first experiment was to disentangle retinotopic (eye-centered) priming from priming that might exist in a more ecologically relevant, non-retinotopic reference frame. We found robust priming in what we labeled to be "spatiotopic" coordinates, but our use of "spatiotopic" was really a catch-all term for multiple non-retinotopic reference frames, including world-centered, object-centered, head-centered, body-centered, and more. In Experiment 2, we attempt to further disentangle these coordinates, namely by separating object-centered coordinates from the "spatiotopic" reference frame. By object-centered, we refer to the target's relative location within the stimulus array. ${ }^{2}$

Object-centered coordinates are highly ecologically relevant, since objects of interest in the real world often move. A sizeable body of research has shown that we represent behaviorally relevant information in both space-based and objectbased coordinates (e.g., Egly, Driver \& Rafal, 1994; Tipper, Weaver, Jerreat \& Burak, 1994; see Chen, 2012, for a review). Specific evidence for object-centered representations has been shown in studies of inhibition of return (IOR: Gibson \& Egeth,

\footnotetext{
${ }^{2}$ The term "object-centered" has been used previously to refer to relative positions either within a single object (e.g., Marr \& Nishihara, 1978; see also Berhmann \& Moscovitch, 1994; Gibson \& Egeth, 1994), or within a larger object structure composed of multiple perceptually grouped parts (e.g., Behrmann \& Tipper, 1994; Tipper, Jordan \& Weaver, 1999). In our study, we are using the term in a way that is conceptually similar to the latter of these two descriptions. That is, in our first experiment, we can consider the six-item array to form a grouped object structure, and we thus favor the term "object-centered" to refer to relative positions within the array. This usage is consistent with Maljkovic and Nakayama's labeling (1996; Experiment 3).
}

1994; Tipper, Jordan \& Weaver, 1999), exogenous facilitation (Lin, 2013), endogenous cueing (Umiltà, Castiello, Fontana, \& Vestri, 1995), express saccades (Edelman, Kristjánsson \& Nakayama, 2007), and visual neglect patients (Behrmann \& Tipper, 1994; Tipper \& Behrmann, 1996).

Several studies have previously addressed whether spatial priming might occur in object-centered coordinates, with some finding evidence for it and others not. Maljkovic and Nakayama (1996, Experiment 3) moved their search arrays across multiple positions from trial to trial, while presenting runs of trials maintaining the target's object-centered location within the array. Results showed an advantage for arraycentered repetitions.

Kristjánsson, Mackeben, and Nakayama (2001) reached the same conclusion using a different task; they presented a "cue" array containing two parallel horizontal lines, within which a target appeared at different relative locations. The location of the cue array was varied unpredictably on each trial, but subjects showed a clear benefit when the target was repeated in the same array-centered location.

Lin and He (2012, Experiment 2) reported yet another approach that could be taken as evidence of object-centered priming. They presented a rectangular frame, in which two "prime" letters were then presented. Subsequently, another frame was presented in an adjacent portion of the display, containing a single target, whose relative position within the frame matched the relative position of one of the primes in the initial frame. Performance was better when the prime in the same "framecentered" position matched the target than when it did not.

Ball and colleagues $(2009,2010,2011)$ conducted a series of studies aimed at dissociating multiple forms of nonretinotopic priming, and within these studies they did not find strong evidence for object-centered priming. In one experiment (Ball et al., 2010), the researchers moved the target location and eye position from trial to trial and employed four types of spatial target repetitions: (1) an "allocentric" condition in which the target always appeared in the same location relative to a salient landmark, whose position was varied from trial to trial (this, in some ways is comparable to an "object-centered" reference frame, in that it is neither retinotopic nor world-centered); (2) an "egocentric-body" condition in which the target always appeared in the same world-centered coordinates (which we would call "spatiotopic"); (3) an "egocentric-eyes" condition in which the target always appeared in the same position relative to fixation (which we would call "retinotopic"); and (4) an "egocentric-combined" condition, in which targets always appeared in the same spatiotopic and retinotopic positions. Similar to Maljkovic and Nakayama (1996), Ball et al. presented each of these priming conditions in runs of seven trials (five target present and two target absent, randomly mixed). There were no runs of control trials. In the "immediate priming" analysis, the RTs to the first and second targets within each run were compared, revealing a significant 


\section{Experiment 2}
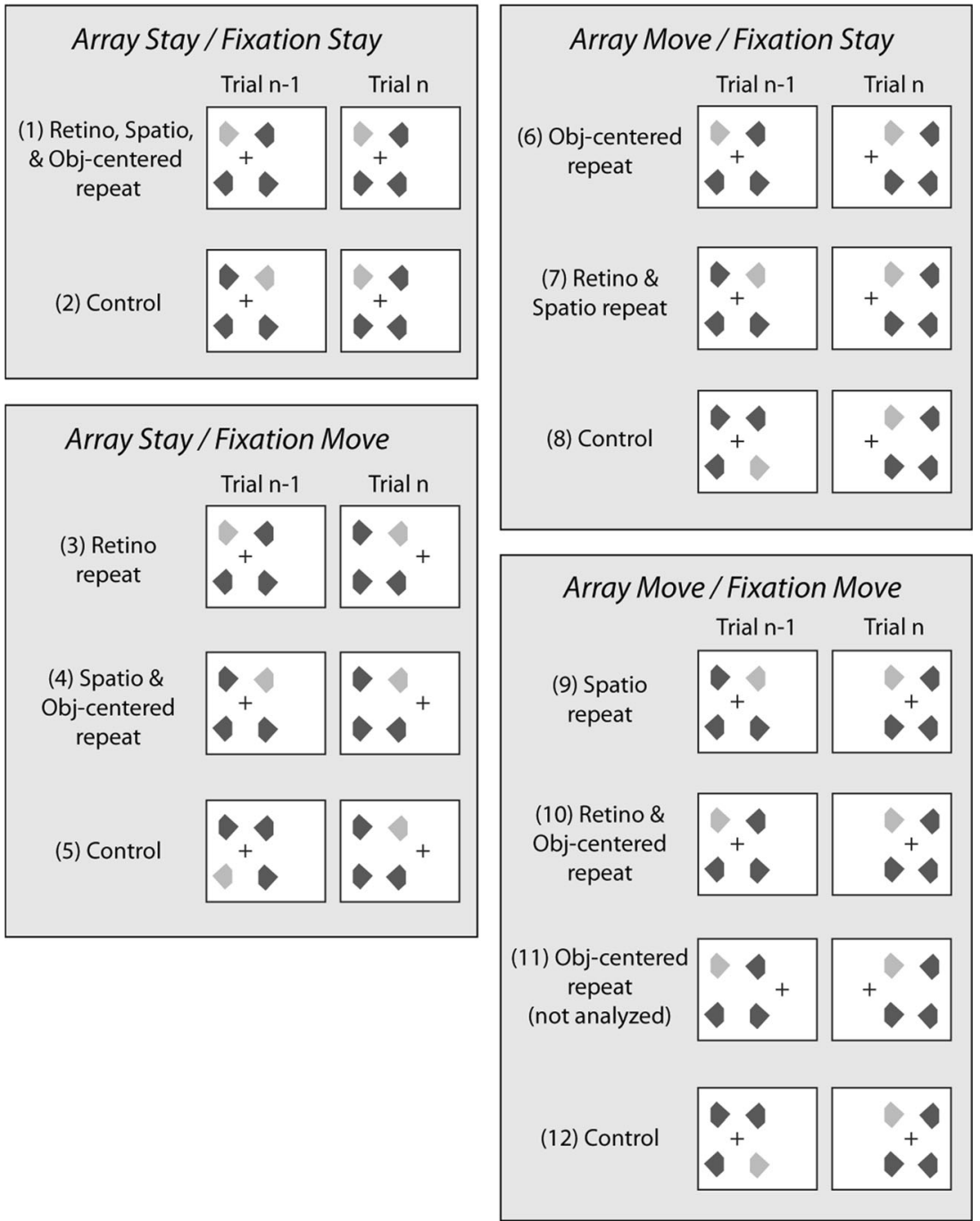

Fig. 3 Representation of stimulus conditions used in Experiment 2, grouped by whether the four-item object array moved between the $n-1$ and $\mathrm{n}$ trial and also by whether the fixation moved between the $n-1$ and $n$

trial. Note that each of these rows depicts just one sample configuration out of many possible per condition; see Method for additional details

RT advantage only in the egocentric-combined condition (i.e., trials with joint retinotopic and spatiotopic repeats). In their second analysis, the researchers examined cumulative priming effects across the five target repetitions of each condition. Here, RT improvement was found in both the egocentricbody (spatiotopic) and egocentric-combined conditions (joint retinotopic and spatiotopic), but there was not reliable allocentric (object-centered) priming (Ball et al., 2010). The authors did find significant cumulative allocentric priming in a previous experiment using a similar procedure (Ball et al., 2009), but the effect was modest and significantly smaller than in their egocentric conditions.

Why do some studies seem to show robust objectcentered priming while others do not? There are several factors to consider. First, in the experiments by Ball and colleagues, the researchers preceded each run of trials with a 3-s break, which could have yielded different levels of attentional readiness for the first and second targets, as measured in their immediate priming analysis. Second, in all of the papers reviewed here, with the exception of the study by Lin \& He (2012), repetitions were presented in runs of trials, so observers could have employed strategic goal-directed attention. Thus, while all of these experiments contribute great insights into understanding attention within multiple spatial reference frames, they might not have afforded pure estimates of spatial priming.

While we have emphasized the importance of removing any potential goal-driven effects when assessing priming, some work suggests this might not matter, arguing 
Table 2 Condition characteristics and results from Experiment 2. Retino retinotopic, spatio spatiotopic, object-cent object-centered, $R T$ response time, $m s$ milliseconds

\begin{tabular}{|c|c|c|c|c|c|c|}
\hline Condition & $\begin{array}{l}\text { Trials/block predicted by } \\
\text { chance }^{\text {a }}\end{array}$ & $\begin{array}{l}\text { Actual range of trials/ } \\
\text { block }^{\mathrm{a}}\end{array}$ & $\begin{array}{l}\mathrm{RT} \\
(\mathrm{ms})\end{array}$ & $\begin{array}{l}\text { Error } \\
(\%)\end{array}$ & $\begin{array}{l}\text { RT priming } \\
(\mathrm{ms})^{\mathrm{b}}\end{array}$ & $\begin{array}{l}\text { Accuracy priming } \\
(\%)^{\mathrm{b}}\end{array}$ \\
\hline \multicolumn{7}{|l|}{ Array stay/fixation stay } \\
\hline $\begin{array}{l}\text { (1) Retino, spatio, and object-cent } \\
\text { repeat }\end{array}$ & 6.75 & $5-9$ & 586 & 2.82 & $39 *$ & 0.80 \\
\hline (2) Control & 15.75 & Not constrained & 625 & 3.62 & - & - \\
\hline \multicolumn{7}{|l|}{ Array stay/fixation move } \\
\hline (3) Retino repeat & 4.5 & $4-7$ & 616 & 2.62 & 7 & 1.88 \\
\hline (4) Spatio, and object-cent repeat & 4.5 & $4-7$ & 590 & 2.64 & $33 *$ & 1.86 \\
\hline (5) Control & 9 & Not constrained & 623 & 4.50 & & \\
\hline \multicolumn{7}{|l|}{ Array move/fixation stay } \\
\hline (6) Object-cent repeat & 4.5 & $4-7$ & 612 & 2.10 & $14^{*}$ & 1.26 \\
\hline (7) Retino and spatio repeat & 4.5 & $4-7$ & 604 & 3.65 & $22 *$ & -0.29 \\
\hline (8) Control & 9 & Not constrained & 626 & 3.36 & - & - \\
\hline \multicolumn{7}{|l|}{ Array move/fixation move } \\
\hline (9) Spatio repeat & 4.5 & $4-7$ & 600 & 3.47 & $22 *$ & 0.44 \\
\hline (10) Retino and object-cent repeat & 4.5 & $4-7$ & 602 & 2.01 & $20 *$ & 1.90 \\
\hline (11) Object-cent repeat ${ }^{\mathrm{c}}$ & 0 & 0 & - & - & - & - \\
\hline (12) Control & 13.5 & Not constrained & 622 & 3.91 & - & - \\
\hline
\end{tabular}

*Significant at alpha $=0.05$ after Holm-Bonferroni correction for multiple comparisons

${ }^{\text {a }}$ Trials per block values were computed for conditions in which both previous and current target were adjacent to fixation—see Experiment 2 method for details

${ }^{\mathrm{b}}$ Priming was defined as the difference between the condition and its corresponding control condition (see Experiment 2 Results for details), and thus priming measures were not applicable to control conditions

${ }^{\mathrm{c}}$ Because of the nature of this condition, it was not possible to have any trials in which both previous and current targets were adjacent to fixation

that goal-driven effects are negligible. Kristjánsson et al. (2001) predictably alternated the target between two object-centered locations, which allowed for goal-driven attention but not spatial priming. They found that RTs were no faster in these alternation runs than in purely random runs of trials, concluding that goal-driven attention, even when possible, should not measurably contribute to the priming effect when target repetitions are predictable. However, the runs of strict alternation were mixed with random runs, so it is unclear if participants were aware that the strict alternations were occurring and/ or able to strategically take advantage of them. In another study, Kristjánsson and Nakayama (2003) measured their participants' explicit knowledge of the repetitions. Even when observers were unaware of such runs of trials, they showed the object-centered advantage. This also suggests such priming could exist outside of goal-driven control. Nevertheless, while it is possible that goal-driven influences only have a small effect on priming, it remains critical to be able to account for these influences within each experiment and determine whether and how they contribute to pure estimates of priming.
In our Experiment 2, we investigated whether nonretinotopic priming, as seen in our Experiment 1, is driven by object-centered and/or spatiotopic priming. The goal was to provide independent measures of multiple forms of priming-retinotopic, spatiotopic, and object-centered-within the same experiment. We reduced the search array to four objects that always appeared grouped together in adjacent locations (see Fig. 3). Now the target location, fixation location, and object array could move from trial to trial. Based on the adaptive account, if the mechanism of spatial priming abides by ecologically relevant coordinates, we should expect contributions of spatiotopic and/or object-centered priming, since both world-centered and object-centered coordinates are behaviorally significant.

\section{Method}

\section{Participants}

Twenty-five participants ( 16 female; mean age $=23.72$ years) were included in this experiment. Most participants completed the task in a single session, but three participants needed to 


\section{Experiment 2}

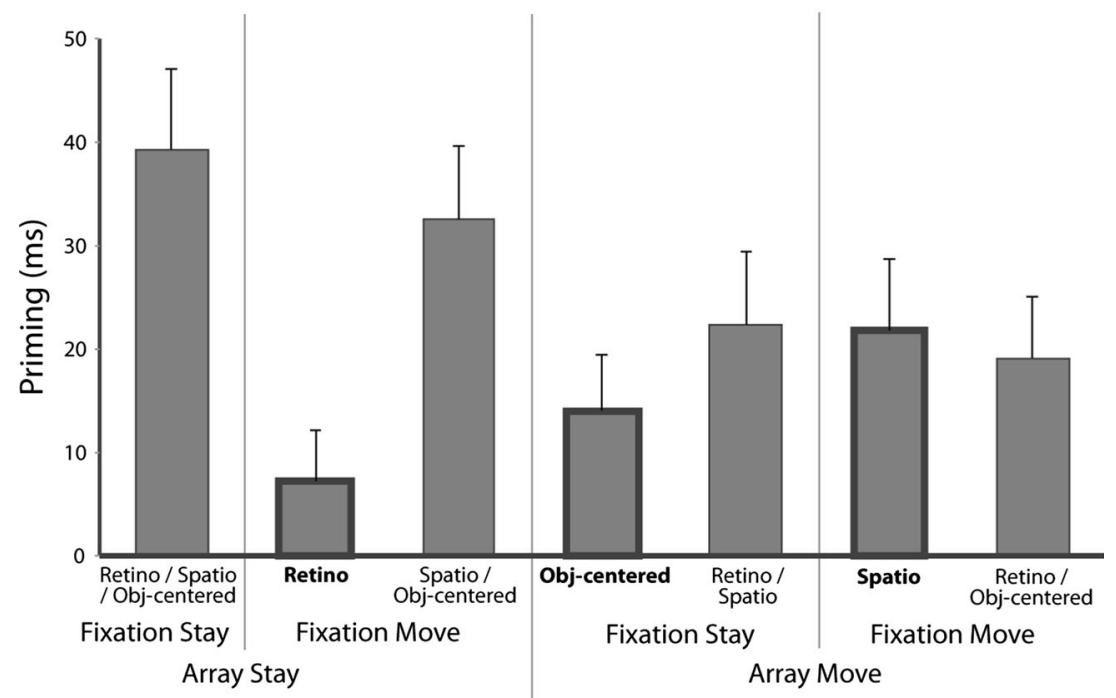

Fig. 4 Results from Experiment 2. Priming is computed as the RT difference between the labeled condition and its corresponding control (see Fig. 3 or Table 2). The critical, pure estimates of priming (Retinotopic, Object-centered, and Spatiotopic) are highlighted in bold. Error bars SEM

return for a second session (and one of these for a third session) to complete the experiment. Three participants failed to reach a minimum criterion of 20 trials per condition after removing fixation and response errors and were not included in the analysis. The recruitment procedures, ethical review, and experiment location were the same as in Experiment 1.

\section{Stimuli and procedure}

Stimuli and procedure were the same as Experiment 1A (horizontal arrangement), with the exception that instead of an

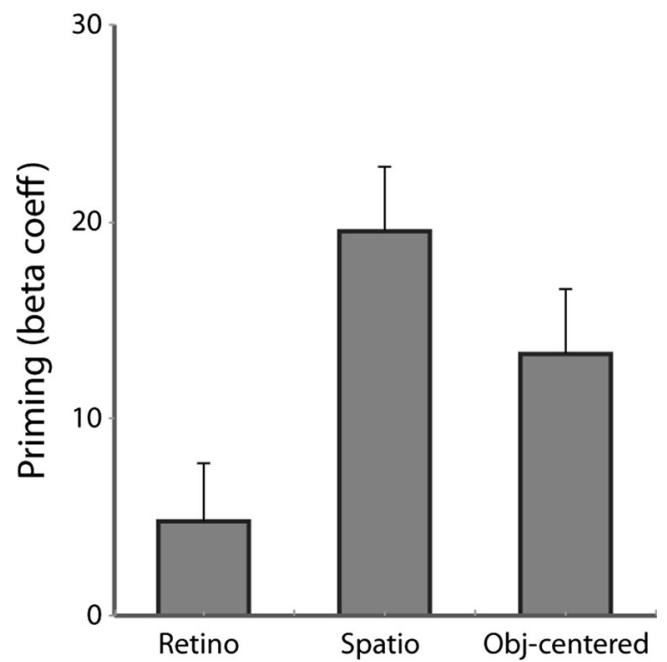

Fig. 5 Experiment 2 regression analysis results. Beta coefficients indicate parameter estimates of priming in Retinotopic, Spatiotopic, and Object-centered coordinates from Model A. See Results for additional details array of six stimuli, only four stimuli were displayed on each trial (See Fig. 3). The four stimuli always included the middle stimulus column from Experiment $1 \mathrm{~A}$ as well as either the left or right columns. As in Experiment 1A, each participant performed the task for $1.5 \mathrm{~h}$, or until they completed eight 144trial blocks, whichever came first.

\section{Design}

Basic target configurations were first generated by crossing two fixation locations, four target locations (i.e., each of the four locations within the object structure), and two object array locations (i.e., whether the group of four items was centered to the left or right side of the display). This yielded 16 target configurations that were each presented 9 times to produce a total of 144 trials per block.

Because of the added variation of object-centered repetitions, Experiment 2 included a total of 12 intertrial conditions (Fig. 3, Table 2).

(1) Array stay/fixation stay/all repeat (array location, fixation location, and target location are all the same on trial $n$ as they were on trial $n-1$; retinotopic, spatiotopic, and object-centered locations all repeat)

(2) Array stay/fixation stay/control (array and fixation locations are the same, but target location is different than in trial $n-1)$

(3) Array stay/fixation move/retino repeat (array location is the same as in trial $n-1$, fixation location is different, and target appears in the same retinotopic location-but 
different spatiotopic and object-centered locations - as in trial $n-1)$

(4) Array stay/fixation move/spatio and object-centered repeat (array location is the same as in trial $n-1$, fixation location is different, and target appears in the same spatiotopic and object-centered location as in trial $n-1$ )

(5) Array stay/fixation move/control (array location is the same as in trial $n-1$, fixation location is different, and target appears in a location that is neither the same retinotopic location nor the same spatiotopic/objectcentered location as in trial $n-1$ )

(6) Array move/fixation stay/object-centered repeat (array location is different from trial $n-1$, fixation location is the same, and target appears in the same object-centered location-but different retinotopic and spatiotopic locations - as in trial $n-1)$

(7) Array move/fixation stay/retino and spatio repeat (array location is different from trial $n-1$, fixation location is the same, and target appears in the same retinotopic/ spatiotopic location as in trial $n-1)$

(8) Array move/fixation stay/control (array location is different from trial $n-1$, fixation location is the same, and target appears in a location that is neither the same object-centered location nor the same retinotopic/ spatiotopic location as in trial $n-1$ )

(9) Array move/fixation move/spatio repeat (array location and fixation location are different from trial $n-1$, and target appears in the same spatiotopic location-but different retinotopic and object-centered locations - as in trial $n-1)$

(10) Array move/fixation move/retino and object-centered repeat (array location and fixation location are different from trial $n-1$, and target appears in the same retinotopic/object-centered location as in trial $n-1$ )

(11) Array move/fixation move/object-centered repeat (array location and fixation location are different from trial $n-1$, and target appears in the same object-centered location as in trial $n-1$. This condition only occurs if the fixation location is located outside the array and the target on trial $n$ or $n-1$ is located at a larger eccentricity; thus, no trials from this condition were actually included in analyses)

(12) Array move/fixation move/control (array location and fixation location are different from trial $n-1$, and target appears in a location that is neither the same spatiotopic location, retinotopic/object-centered location, nor object-centered location as in trial $n-1)$.

As in Experiment 1, the ordering of these trials was generated to ensure a sufficient number of trials in each of the critical conditions. We performed an iterative randomization procedure to produce a sequence of trials that conformed to the following constraints: exactly $50 \%$ of trials were fixation stay and $50 \%$ were fixation move, exactly $50 \%$ of trials were array stay and $50 \%$ were array move, no more than five trials in a row contained the same fixation location, no more than five trials in a row contained the same object-centered location. As before, we included a constraint to ensure that the lower frequency conditions would have a minimum number of trials per block, as indicated in Table 2. Because we were restricting the analysis to close-eccentricity trials, we applied the constraints on the number of trials per condition only to those trials for which previous and current targets were close in eccentricity. Note that it was impossible for the array move/ fixation movelobject-centered repeat condition to have trials in which both the $n-1$ and current targets were close in eccentricity. We therefore did not constrain this condition in the randomization procedure and did not intend to analyze it.

\section{Results and discussion}

\section{Overall performance}

Mean fixation accuracy was $83.17 \%(\mathrm{SD}=10.15 \%)$, and all trials with fixation errors were excluded from further analysis. Overall response accuracy was $96.29 \%(\mathrm{SD}=3.07 \%)$. RT data were trimmed at $3 \mathrm{SD}$ above each participant's mean, separately for trials that were close in eccentricity (i.e., adjacent to fixation) and far (non-adjacent). Overall trimmed RTs were faster for close-eccentricity trials than far-eccentricity trials, $614 \mathrm{~ms}$ and $699 \mathrm{~ms}$ respectively, $t(21)=11.86, P<$ 0.001 . The remaining analysis is focused on trials in which both $n-1$ and current target locations were close in eccentricity.

\section{Spatial priming effects}

Reaction time Mean RTs for each of the 12 intertrial conditions are reported in Table 2. The seven critical "repeat" conditions were compared to their respective control conditions to yield distinct spatial priming scores (shown in Fig. 4 and Table 2). As in Experiment 1, we used paired samples $t$-tests with a Holm-Bonferroni correction to test the priming scores for significance. We will place special emphasis in interpreting the results of pure retinotopic priming, pure object-centered priming, and pure spatiotopic priming (labeled below as 2, 4, and 6, respectively).

(1) Retino/Spatio/Obj-Centered Priming (Array \& Fixation Stay). When both the fixation and the array stayed constant, repeated targets shared the same retinotopic, spatiotopic, and object-centered locations. Priming was greatest in this condition, and significant compared to its control, $t(21)=4.99, P_{\text {adjusted }}=0.00043, d=1.06$ 
(2) Retino Priming (Array Stay, Fixation Move). By moving the fixation position but keeping the array position constant between trials, we could isolate retinotopic priming from the other coordinates. Results failed to show significant priming for pure retinotopic repeats, $t(21)=1.44$, $P_{\text {adjusted }}=0.162 d=0.31$.

(3) Spatio/Obj-Centered Priming (Array Stay, Fixation Move). This comparison was functionally the same as the "spatiotopic" condition of Experiment 1; because the array was held constant, this priming contains contributions of both spatiotopic and object-centered coordinates. Results showed significant priming, $t(21)=4.54$, $P_{\text {adjusted }}=0.0011, d=1.06$, replicating Experiment 1 .

(4) Object-Centered Priming (Array Move, Fixation Stay). This is the estimate of pure object-centered priming, and results were significant, $t(21)=2.57, p_{\text {adjusted }}=0.0036, d$ $=0.55$.

(5) Retino/Spatio Priming (Array Move, Fixation Stay). We also obtained significant priming for the combined retinotopic/spatiotopic (but not object-centered) measure, $t(21)=3.16, P_{\text {adjusted }}=0.014, d=0.67$.

(6) Spatiotopic Priming (Array Move, Fixation Move). Our estimate of pure spatiotopic priming was significant, $t(21)=3.18, P_{\text {adjusted }}=0.018, d=0.68$.

(7) Retino/Obj-Centered Priming (Array Move, Fixation Move). We observed significant priming in this comparison, $t(21)=3.21, P_{\text {adjusted }}=0.021, d=0.68$.

Replicating Experiment 1A, we failed to find significant retinotopic priming but did find significant spatio/object centered priming (which is functionally the same as "spatiotopic" from Experiment 1A). We were further able to disentangle spatiotopic and object-centered measures and observed robust priming in both. We compared the three pure priming scores (retinotopic, spatiotopic, and object-centered) via a 3-level one-way ANOVA; this test did not reach significance, $F(2$, 42) $=1.729, p=0.190, \eta_{\mathrm{p}}{ }^{2}=.076$.

Regression analysis In order to take advantage of the whole data set, we employed multiple linear regression on all 11 intertrial conditions, allowing us to extract more stable estimates of the three pure forms of priming (Model A), as well as potential interactions between them (Models B and C).

For each participant, we constructed a basic regression model (Model A) containing five categorical predictors and one dependent variable (RT). The predictors were as follows: array repetition, fixation repetition, retinotopic repetition, spatiotopic repetition, and object-centered repetition (plus an additional constant term). On each trial, the weight assigned to each predictor depended on repeat vs. switch from the $n-1$ trial, where a " 0 " was assigned for repeats and " 1 " was assigned for switches. For example, a trial in the Array Move / Fixation Stay / Retino and Spatio Repeat condition would be assigned the five respective predictor weights as follows: $1,0,0,0$, and 1 .

We obtained beta coefficients for our three predictors of interest: retinotopic repetition, spatiotopic repetition, and object-centered repetition. These coefficients estimated the three respective priming effects, and are plotted in Fig. 5. Each of these mean betas was tested for significance against a hypothesized mean of 0 , using two-tailed t-tests with a Holm-Bonferroni correction. Results were similar to those from the initial RT priming analysis above: retinotopic priming was not significant, $t(21)=1.59, P_{\text {adjusted }}=0.128, d=$ 0.34 ; spatiotopic priming was significant, $t(21)=6.04$, $P_{\text {adjusted }}=0.00002, d=1.29$; object-centered priming was significant, $t(21)=4.16, P_{\text {adjusted }}=0.0009, d=0.89$. Notably, when compared to the initial RT priming analysis, the effect size for retinotopic priming was virtually the same, but the effect sizes for spatiotopic and object-centered priming grew substantially, reflecting the increased statistical power of the model.

We entered the three sets of betas (for the retino, spatio, and object-centered priming) into a three-level one-way ANOVA to determine whether they significantly varied from one another, and this test was significant, $F(2,42)=5.179$, $P=0.010, \eta_{\mathrm{p}}{ }^{2}=.198$. Holm-Bonferroni corrected two-tailed post-hoc comparisons showed that spatio priming was significantly greater than retino priming, $t(21)=3.23$, $P_{\text {adjusted }}=0.012, d=0.69$. However, object-centered priming was not significantly different than retino priming, although the uncorrected $P$-value on this test was marginal, $t(21)=1.91, P_{\text {uncorrected }}=0.069, P_{\text {adjusted }}=0.139, d=0.41$. Additionally, spatio priming was not significantly different from object-centered priming, $t(21)=1.30, P_{\text {adjusted }}=0.208$, $d=0.28$.

We next asked: are these effects additive? The basic regression model described above was focused on obtaining pure estimates of each type of priming and did not test for potential interactions, to maximize statistical power. Having established the pure estimates of priming in Model A, we ran an expanded model in Model B, adding the four interaction terms to the original model: retino $\times$ spatio, retino $\times$ object-centered, spatio $\times$ object-centered, and the 3-way interaction. None of these predictors accounted for any significant variance, but the original predictors (the pure priming estimates) returned slightly less robust results, suggesting that the new model contained too many parameters and possibly lacked power to detect significant interactions. We thus tried a different approach in Model C, which is actually a group of regression models. Here, we added only one interaction term to the model at a time (i.e., we ran four separate regressions, each with the original five parameters plus one of the interaction terms). In these cases, the three pure priming estimates were virtually unchanged from Model A, and critically, none of the interaction terms accounted for any significant variance (all uncorrected $P$ 
values $>0.64)$. Thus, retino, spatio, and object-centered priming appear to be additive.

Accuracy Mean error rates for the 11 intertrial conditions and seven priming scores are reported in Table 2. HolmBonferroni corrected paired-samples $t$-tests did not reveal any significant priming in any of the seven conditions, and a one-way ANOVA was carried out to test for differences among the conditions. Mauchly's test of sphericity revealed unequal variances across the seven conditions, so we applied a Greenhouse-Geisser correction, and results were nonsignificant, $F(3.698,77.661)<1$.

\section{General discussion}

We set out to ask whether spatial priming is adaptive or merely an architectural byproduct of ongoing visual processing. We designed two experiments to isolate distinct types of spatial priming across several reference frames: retinotopic, spatiotopic, and object-centered. The byproduct account predicted that spatial priming would remain, by default, in retinotopic coordinates across eye movements or after the grouped array of elements moved from trial to trial. In contrast, the adaptive account predicted that observers would implement an additional, non-compulsory step to update their spatial priority maps in between trials in order to carry the priming benefit forward into ecologically relevant coordinates. The results clearly supported the latter of these two predictions: we saw robust spatiotopic and object-centered priming but did not see consistent retinotopic priming. We thus infer that spatial priming is behaviorally adaptive, designed to maximize performance in our commerce with the world.

Our support for the adaptive account largely agrees with claims originally made by Maljkovic and Nakayama (1996; see also Chun \& Nakayama, 2000; Maljkovic \& Nakayama, 2000) and goes further by producing and comparing pure estimates of three forms of priming while removing the potential contributions of strategic, goal-directed attention. Our findings are also consistent with a recent paper (published after we submitted this paper) by Gokce, Müller, \& Geyer (2015) reporting reliable effects of spatiotopic and objectcentered priming across two experiments (see also a conference abstract from Vaziri Pashkam \& Cavanagh, 2009).

\section{Retinotopic vs. non-retinotopic reference frames}

The non-retinotopic priming observed in this study was driven independently by both spatiotopic (world- or screen-centered) and object-centered (relative position in the stimulus array) effects. It is worth noting that our spatiotopic condition - even after disentangling it from object-centered effects in
Experiment 2-could still reflect contributions from multiple possible coordinate frames, including absolute (i.e., worldcentered) coordinates, screen-centered coordinates (which could also include an object/frame-centered contribution from the outline of the monitor), and head- or body-centered coordinates (see Ball et al., 2010, 2011). Further teasing apart each of these different non-retinotopic contributions would be an interesting avenue for future research, but Experiment 2 demonstrates that at least some component of priming exists in allocentric, object-centered coordinates, while additional priming persists even when this object array changes (pure spatiotopic condition). This co-existence of object-centered and space-based components is consistent with a number of experimental approaches in the larger attention literature, as reviewed in the introduction to Experiment 2 (e.g., Chen, 2012; Egly, et al., 1994; Gibson \& Egeth, 1994; Lin, 2013; Tipper, et al., 1994, 1999; Umiltà et al., 1995). Of note is that many of these studies separating object-centered effects from space-based effects have not tested whether the space-based effects are retinotopic or spatiotopic. In our study, we were able to tease apart these effects by including both object array movements and eye movements, finding that both objectcentered and space-based components of spatial priming can occur in non-retinotopic, ecologically relevant reference frames. The retinotopic vs. non-retinotopic distinction is crucial in testing the adaptive vs. by-product hypotheses for spatial priming.

A great deal of research has explored whether retinotopic and/or spatiotopic (i.e., eye-centered vs. eye position independent) effects are seen for a variety of visual tasks. Results have often been mixed, with retinotopic and spatiotopic effects debated in cases of trans-saccadic integration (Irwin, 1991; McRae, Butler, \& Popiel, 1987; Hayhoe, Lachter, \& Feldman, 1991; Melcher \& Colby, 2008; Burr \& Morrone, 2011), motion and tilt aftereffects (Melcher \& Morrone, 2003; Knapen, Rolfs, \& Cavanagh, 2009; Knapen et al, 2010; Ezzati, Golzar, \& Afraz 2008), inhibition of return (Pertzov, Zohary, \& Avidan, 2010; Mathôt \& Theeuwes, 2010; Hilchey, Klein, Satel, \& Wang, 2012; Satel, Wang, Hilchey \& Klein, 2012), perceptual learning (Zhang \& Li, 2010; Zhang, Zhang \& Li, 2013), and spatial memory (Golomb \& Kanwisher, 2012a; Lin \& Gorea, 2011; Karn, Møller, \& Hayhoe, 1997). While spatiotopic effects are sometimes found behaviorally, there seems to be little evidence for explicit spatiotopic organization in the brain (Golomb \& Kanwisher, 2012b; Gardner, Merriam, Movshon, \& Heeger, 2008; but see Crespi et al., 2011; d'Avossa et al., 2007), and most mechanisms of spatiotopic stability rely on the updating, or remapping, of natively retinotopic maps (Cohen \& Andersen, 2002; Duhamel, Colby, \& Goldberg 1992; Golomb et al, 2008, Golomb, Nguyen-Phuc, et al., 2010, Golomb, Pulido, et al., 2010; Cavanagh, Hunt, Afraz, \& Rolfs, 2010). 
Why do we not also find reliable retinotopic effects in our experiment? One possibility is that trial-to-trial variability in precise eye position (e.g., microsaccades within the $2^{\circ}$ window) could have contributed noise detrimental to retinotopic priming. A more likely reason, however, is that the priming effects we measured occur on a fairly long timescale (a few seconds between trials). On trials that contained eye movements, the stimuli did not appear until the observers had completed the eye movement and maintained fixation for a full second. Thus, we are not looking at the dynamic saccadic updating period right around the time of a saccade, and any residual retinotopic trace would have long decayed by this point (Golomb et al., 2008). Thus, our claim is not that spatial priming "natively" operates in spatiotopic coordinates; we assume that the underlying priority maps are still retinotopic, but that they have had time to update to reflect the more ecologically relevant coordinates following the eye movement. Because priority maps do not always update to spatiotopic coordinates - this step has been shown to depend on task-relevance (Golomb et al., 2008) - the fact that the maps do update in the current study demonstrates the adaptiveness of spatial priming.

\section{If priming is adaptive, why isn't it optimal?}

In considering the adaptive nature of spatial priming, we are left with an unresolved puzzle. Given that, by design, target locations were unpredictable on each trial, priming could not have served any behavioral benefit within the context of these experiments. So, why bias spatial priority to previous target locations? While we cannot provide an answer to this question based on the current data, we entertain three possibilities below, which are not necessarily mutually exclusive.

(1) Spatial priming is usually beneficial. Over a lifetime of experience - or even the evolutionary timescale - it is usually the case that objects of interest remain so for longer than a fleeting moment (as discussed earlier in our examples of drinking coffee and waiting for traffic lights to turn green). Given that priming usually benefits behavior, an efficiently designed visual system might always employ it, absorbing the inefficiencies associated with occasional episodes in which spatial priming is not beneficial (e.g., during an artificially created perception experiment). To build a more efficient kind of priming mechanism requires performance monitoring, such as evaluatory cognitive control mechanisms that have been described by others (e.g., Botvinick, Braver, Barch, Carter \& Cohen, 2001; Kerns, Cohen, MacDonald, Cho, Stenger \& Carter, 2004). However, engaging additional cognitive processes is likely to carry a metabolic cost (e.g., Muraven \& Baumeister, 2000), and for a brain that needs to manage energy demands, such monitoring might not be worth the added costs. Interestingly, recent work has shown greater spatial biasing toward recent target locations that were associated with high reward, suggesting that some form of evaluation could modulate spatial priming effects (Hickey, Chelazzi, \& Theeuwes, 2010; see also Camara, Manohar \& Husain, 2013). However, it is not clear whether the reward effects are additive or interactive with priming, and future work will be needed to address this question.

(2) Spatial priming forms the building blocks of probability cueing. Spatial priming has been conceptualized by others as a mechanism that decays within minutes, so that it is principally useful for relatively brief episodes in which we repeatedly interact with a current object of interest (Maljkovic \& Nakayama, 2000). However, some researchers have posited a longer-term role for spatial priming, specifically claiming that it forms the building blocks of probability cueing (Walthew \& Gilchrist, 2006), in which stable locations of interest in the environment gain prolonged prioritization. If Walthew and Gilchrist are correct (but see Druker \& Anderson, 2010; Geng \& Behrmann, 2005; Jiang, Swallow, Rosenbaum \& Herzig, 2013, for debate on this point), it is plausible that the purpose of spatial priming is to constantly increment spatial priority at recent locations of interest in space, ultimately producing the long-term biases when spatial statistical regularities are present. This idea is consistent with instance-based theories of learning (Logan, 1988), in which learning becomes stronger over time because with each instance a separate memory trace is laid down that can be recruited at retrieval.

If spatial priming and probability cueing are related, we might expect the two phenomena to behave similarly across multiple reference frames. Jiang and Swallow (2013a) carried out a probability cueing study where they positioned a monitor such that its surface was parallel to the floor, and had participants move among multiple different vantage points from trial to trial to perform the task. Results showed probability cueing only when rich portions of the display were maintained in egocentric coordinates; when the rich portions were held in allocentric coordinates, no learning was observed (see also Jiang \& Swallow, 2013b; Jiang, Swallow \& Capistrano, 2013; Jiang, Swallow \& Sun, 2014).

One might question why probability cueing would not occur in allocentric coordinates, given that it is assumed to serve a behaviorally adaptive purpose. Jiang, Swallow, and Capistrano (2013) reasoned that learning often happens from a consistent viewpoint, in environments with constrained structures, such that an egocentric learning mechanism would be more useful. Moreover, these results may be dependent on the 
environmental scale, as probability cueing has been observed in allocentric coordinates when tested in larger, relatively unconstrained, real-world environments (Jiang, Won, Swallow, \& Mussack, 2014; Smith, Hood \& Gilchrist, 2008; 2010). Note that these experiments are not directly comparable to the present work, because we held body/head position constant, and one could imagine that priming may be dependent on the observer maintaining a single position. Ball et al. (2011) included a head-centered manipulation, using two adjacent monitors and asking participants to move their head position from trial to trial to respond to stimuli on the two respective monitors. They found evidence only for head-centered (i.e., egocentric) priming, analogous to the probability cueing results by Jiang et al. (Jiang \& Swallow, 2013a, 2013b; Jiang, Swallow, \& Capistrano, 2013, Jiang, Swallow, Rosenbaum, et al., 2013, Jiang, Swallow, \& Sun, 2014, Jiang, Won, et al., 2014). However, it would be important to test whether the same results emerge with unpredictable target locations.

(3) Spatial priming forms the building blocks of contextual cueing. Much like some researchers have argued that spatial priming underlies probability cueing, others have linked spatial priming to contextual cueing (although Chun and Nakayama argued that the two are "distinct, independent mechanisms"; Chun \& Nakayama, 2000, p. 77). Geyer et al. (2007) pointed out that previous studies of spatial priming used the same display configuration on each trial (i.e., three objects, each forming the vertex of an invisible equilateral triangle). Thus, trials containing spatial repetitions were also trials in which the target location was repeated within a spatial configuration. In this way, cumulative spatial priming could serve as the building blocks of long-term contextual cueing, consistent with instance-based learning (Logan, 1988). By this account, priming only lasts for roughly eight trials - in contrast to the long-term effects of contextual cueing-because a stable association between the spatial configuration and a specific target location never develops. A complementary prediction, made by Geyer et al., is that priming would suffer if the spatial configurations were varied from trial to trial, which is indeed what they found, supporting a linkage between the two spatial phenomena (Geyer et al, 2007; see also Gokce, Müller, \& Geyer, 2013). If one considers configuration repetitions as repetitions of an object structure, this study also lends support for spatial priming occurring within an object-centered frame of reference, although this condition is more analogous to our combined retino/spatio/object-centered priming condition.

\section{Related issues: feature priming and spatial inhibition}

The present work raises a number of questions that are ripe for future investigation. First, while we focused on spatial priming, similar priming of popout effects have been found for feature repetitions (Maljkovic \& Nakayama, 1994). Featurebased priming has been studied much more extensively in the literature than spatial priming, and numerous theories of its function have been proposed (Bichot \& Schall, 2002; Fecteau \& Munoz, 2003; Fecteau, 2007; Lamy, Yashar \& Ruderman, 2010; Kristjánsson \& Campana, 2010; Maljkovic \& Nakayama, 1994; 2000). Although the present study did not have the necessary statistical power to compare feature-based (color) priming during retinotopic, spatiotopic, and objectcentered repetitions specifically, a basic analysis confirmed that robust color-based priming was present overall, even across changes in eye position (e.g., in Experiment 2, color priming was $29 \mathrm{~ms}$ on fixation stay trials and $28 \mathrm{~ms}$ on fixation move trials). A 2 (color repetition) $\times 2$ (fixation repetition) ANOVA showed a significant main effect of color repetition, $\mathrm{F}(1,21)=75.972, P<0.00001, \eta_{\mathrm{p}}{ }^{2}=.783$., while neither the main effect of fixation repetition nor the 2-way interaction was significant, both $F s<1$.

Second, while our study focused on the facilitatory benefits of spatial target repetitions across multiple reference frames, we did not discuss possible inhibitory effects. As mentioned in the introduction, several studies have examined such inhibitory effects (e.g., Maljkovic \& Nakayama, 1996; Geyer et al., 2007; 2010), and it would be interesting to contrast them across the reference frames. However, measuring inhibitory priming requires a control condition in which the target on the current trial is placed in a location that was empty in the previous trial, and we did not design our stimuli to allow for this (but see the recent work by Gokce et al., 2015, who did examine inhibitory priming across multiple reference frames and found exclusively object-centered effects).

\section{Final remarks}

In this study, spatial priming has passed a critical test of ecological validity. We thus add it to a diverse series of mechanisms by which our past experiences guide how we spatially prioritize the world. It will be essential to understand how each of these mechanisms relate to one another, and further manipulations of spatial reference frames promise to continue shedding light on this pursuit.

Acknowledgments Portions of this work were presented at the Twelfth Annual Meeting of the Vision Sciences Society (Naples, FL) in May 2012. Support was provided by National Science Foundation- Behavioral \& Cognitive Sciences (NSF BCS) grant 1027054 to A. B. L., the United States-Israel Binational Science Foundation (US-Israel BSF) grant 2009424 to A. B. L., and National Institutes of Health (NIH) F32EY020157 to J. D. G. We thank Arni Kristjánsson and two anonymous 
reviewers for helpful comments and suggestions. We also thank Nancy Kanwisher for additional research support, Aude Oliva for sharing her laboratory's eye-tracking setup, and Elana Ben-Akiva for assistance in running participants

\section{References}

Anderson, B. A. (2014). Value-driven attentional capture is modulated by spatial context. Visual Cognition, 23(1-2), 67-81.

Anstis, S., Verstraten, F. A., \& Mather, G. (1998). The motion aftereffect. Trends in Cognitive Sciences, 2(3), 111-117.

Ball, K., Lane, A., Ellison, A., \& Schenk, T. (2011). Spatial priming in visual search: Memory for body-centered information. Experimental Brain Research, 212(3), 477-485.

Ball, K., Smith, D., Ellison, A., \& Schenk, T. (2009). Both egocentric and allocentric cues support spatial priming in visual search. Neuropsychologia, 47, 1585-1591.

Ball, K., Smith, D., Ellison, A., \& Schenk, T. (2010). A body-centered frame of reference drives spatial priming in visual search. Experimental Brain Research, 204(4), 585-594.

Becker, S. I. (2008). The stage of priming: Are intertrial repetition effects attentional or decisional? Vision Research, 48(5), 664-684.

Behrmann, M., \& Moscovitch, M. (1994). Object-centered neglect in patients with unilateral neglect: Effects of left-right coordinates of objects. Journal of Cognitive Neuroscience, 6(1), 1-16.

Berhmann, M., \& Tipper, S. P. (1994). Object-based attentional mechanisms: Evidence from patients with unilateral neglect. In C. Urnilta $\&$ M. Moscovitch (Eds.), Attention and performance XIV conscious and unconscious processing and cognitive functioning (pp. 351375). Hillsdale, NJ: Erlbaum.

Bichot, N. P., \& Schall, J. D. (2002). Priming in macaque frontal cortex during popout visual search: Feature-based facilitation and locationbased inhibition of return. The Journal of Neuroscience, 22(11), 4675-4685.

Bisley, J. W., \& Goldberg, M. E. (2010). Attention, intention, and priority in the parietal lobe. Annual Review of Neuroscience, 33, 1.

Botvinick, M. M., Braver, T. S., Barch, D. M., Carter, C. S., \& Cohen, J. D. (2001). Conflict monitoring and cognitive control. Psychological Review, 108(3), 624

Brainard, D. H. (1997). The psychophysics toolbox. Spatial Vision, 10, 433-436.

Burr, D. C., \& Morrone, M. C. (2011). Spatiotopic coding and remapping in humans. Philosophical Transactions of the Royal Society, B: Biological Sciences, 366(1564), 504-515.

Camara, E., Manohar, S., \& Husain, M. (2013). Past rewards capture spatial attention and action choices. Experimental Brain Research, 230(3), 291-300.

Cavanagh, P., Hunt, A. R., Afraz, A., \& Rolfs, M. (2010). Visual stability based on remapping of attention pointers. Trends in Cognitive Sciences, 14(4), 147-153.

Chelazzi, L., Estocinova, J., Calletti, R., Lo Gerfo, E., Sani, I., Libera, C. D., \& Santandrea, E. (2014). Altering spatial priority maps via reward-based learning. The Journal of Neuroscience, 34(25), 8594-8604

Chen, Z. (2012). Object-based attention: A tutorial review. Attention, Perception, \& Psychophysics, 74(5), 784-802.

Chun, M. M. (2000). Contextual cueing of visual attention. Trends in Cognitive Sciences, 4(5), 170-178.

Chun, M. M., \& Jiang, Y. (1998). Contextual cueing: Implicit learning and memory of visual context guides spatial attention. Cognitive Psychology, 36, 28-71.
Chun, M. M., \& Nakayama, K. (2000). On the functional role of implicit visual memory for the adaptive deployment of attention across scenes. Visual Cognition, 7, 65-81.

Cohen, Y. E., \& Andersen, R. A. (2002). A common reference frame for movement plans in the posterior parietal cortex. Nature Reviews Neuroscience, 3(7), 553-562.

Crespi, S., Biagi, L., d'Avossa, G., Burr, D. C., Tosetti, M., \& Morrone, M. C. (2011). Spatiotopic coding of BOLD signal in human visual cortex depends on spatial attention. PLoS One, 6(7), e21661.

d'Avossa, G., Tosetti, M., Crespi, S., Biagi, L., Burr, D. C., \& Morrone, M. C. (2007). Spatiotopic selectivity of BOLD responses to visual motion in human area MT. Nature Neuroscience, 10(2), 249-255.

Druker, M., \& Anderson, B. (2010). Spatial probability aids visual stimulus discrimination. Frontiers in Human Neuroscience, 4.

Duhamel, J.-R., Colby, C. L., \& Goldberg, M. E. (1992). The updating of the representation of visual space in parietal cortex by intended eye movements. Science, 255(5040), 90-92.

Edelman, J. A., Kristiánsson, Á., \& Nakayama, K. (2007). The influence of object-relative visuomotor set on express saccades. Journal of Vision, 7(6), 12.

Egly, R., Driver, J., \& Rafal, R. D. (1994). Shifting visual attention between objects and locations: Evidence from normal and parietal lesion subjects. Journal of Experimental Psychology: General, 123(2), 161.

Ezzati, A., Golzar, A., \& Afraz, A. S. (2008). Topography of the motion aftereffect with and without eye movements. Journal of Vision, $8(14), 23$.

Fecteau, J. H. (2007). Priming of pop-out depends upon the current goals of observers. Journal of Vision, 7(6), 1.

Fecteau, J. H., \& Munoz, D. P. (2003). Exploring the consequences of the previous trial. Nature Reviews Neuroscience, 4(6), 435-443.

Fiser, J., \& Aslin, R. N. (2001). Unsupervised statistical learning of higher-order spatial structures from visual scenes. Psychological Science, 12, 499-504.

Gardner, J. L., Merriam, E. P., Movshon, J. A., \& Heeger, D. J. (2008). Maps of visual space in human occipital cortex are retinotopic, not spatiotopic. The Journal of Neuroscience, 28(15), 3988-3999.

Geng, J. J., \& Behrmann, M. (2002). Probability cuing of target location facilitates visual search implicitly in normal participants and patients with hemispatial neglect. Psychological Science, 13(6), 520-525.

Geng, J. J., \& Behrmann, M. (2005). Spatial probability as an attentional cue in visual search. Perception \& Psychophysics, 67(7), 12521268.

Geyer, T., \& Müller, H. J. (2009). Distinct, but top-down modulable color and positional priming mechanisms in visual pop-out search. Psychological Research, 73, 167-176.

Geyer, T., Müller, H. J., \& Krummenacher, J. (2007). Cross-trial priming of element positions in visual pop-out search is dependent on stimulus arrangement. Journal of Experimental Psychology: Human Perception and Performance, 33(4), 788-797.

Geyer, T., Zehetleitner, M., \& Müller, H. J. (2010). Positional priming of pop-out: A relational-encoding account. Journal of Vision, 10(2):3, $1-17$.

Gibson, B. S., \& Egeth, H. (1994). Inhibition of return to object-based and environment-based locations. Perception \& Psychophysics, 55(3), 323-339.

Gokce, A., Müller, H. J., \& Geyer, T. (2013). Positional priming of popout is nested in visuospatial context. Journal of Vision, 13(3), 32.

Gokce, A., Müller, H. J., \& Geyer, T. (2015). Positional priming of visual pop-out search is supported by multiple spatial reference frames. Frontiers in Psychology, 6. doi:10.3389/fpsyg.2015.00838

Golomb, J. D., Chun, M. M., \& Mazer, J. A. (2008). The native coordinate system of spatial attention is retinotopic. Journal of Neuroscience, 28(42), 10654-10662.

Golomb, J. D., \& Kanwisher, N. (2012). Retinotopic memory is more precise than spatiotopic memory. Proceedings of the National 
Academy of Sciences of the United States of America, 109(5), 17961801.

Golomb, J. D., \& Kanwisher, N. (2012). Higher level visual cortex represents retinotopic, not spatiotopic, object location. Cerebral Cortex, 22(12), 2794-2810.

Golomb, J. D., L'Heureux, Z. E., \& Kanwisher, N. (2014). Featurebinding errors after eye movements and shifts of attention. Psychological Science, 25(5), 1067-1078.

Golomb, J. D., Marino, A. C., Chun, M. M., \& Mazer, J. A. (2011). Attention doesn't slide: Spatiotopic updating after eye movements instantiates a new, discrete attentional locus. Attention, Perception, \& Psychophysics, 73(1), 7-14.

Golomb, J. D., Nguyen-Phuc, A. Y., Mazer, J. A., McCarthy, G., \& Chun, M. M. (2010). Attentional facilitation throughout human visual cortex lingers in retinotopic coordinates after eye movements. The Journal of Neuroscience, 30(31), 10493-10506.

Golomb, J. D., Pulido, V. Z., Albrecht, A. R., Chun, M. M., \& Mazer, J. A. (2010). Robustness of the retinotopic attentional trace after eye movements. Journal of Vision, 10(3), 1-12.

Hayhoe, M., Lachter, J., \& Feldman, J. (1991). Integration of form across saccadic eye movements. Perception, 20(3), 393-402.

Hickey, C., Chelazzi, L., \& Theeuwes, J. (2010). Reward changes salience in human vision via the anterior cingulate. Journal of Neuroscience, 30(33), 11096-11103.

Hilchey, M. D., Klein, R. M., Satel, J., \& Wang, Z. (2012). Oculomotor inhibition of return: How soon is it "recoded" into spatiotopic coordinates? Attention, Perception, \& Psychophysics, 74(6), 1145-1153.

Hillstrom, A. P. (2000). Repetition effects in visual search. Perception \& Psychophysics, 62(4), 800-817.

Hoffmann, J., \& Kunde, W. (1999). Location-specific target expectancies in visual search. Journal of Experimental Psychology: Human Perception and Performance, 25(4), 1127-1141.

Holm, S. (1979). A simple sequentially rejective multiple test procedure. Scandinavian Journal of Statistics, 6(2), 65-70.

Huang, L., Holcombe, A. O., \& Pashler, H. (2004). Repetition priming in visual search: Episodic retrieval, not feature priming. Memory \& Cognition, 32(1), 12-20.

Irwin, D. E. (1991). Information integration across saccadic eye movements. Cognitive Psychology, 23(3), 420-456.

Jiang, Y. V., \& Swallow, K. M. (2013). Spatial reference frame of incidentally learned attention. Cognition, 126, 378-390.

Jiang, Y. V., \& Swallow, K. M. (2013). Body and head tilt reveals multiple frames of reference for spatial attention. Journal of Vision, 13(13).

Jiang, Y. V., Swallow, K. M., Rosenbaum, G. M., \& Herzig, C. (2013). Rapid acquisition but slow extinction of an attentional bias in space. Journal of Experimental Psychology: Human Perception and Performance, 39(1), 87.

Jiang, Y. V., Swallow, K. M., \& Capistrano, C. G. (2013). Visual search and location probability learning from variable perspectives. Journal of Vision, 13(6), 13.

Jiang, Y. V., Swallow, K. M., \& Sun, L. (2014). Egocentric coding of space for incidentally learned attention: Effects of scene context and task instructions. Journal of Experimental Psychology: Learning, Memory, \& Cognition, 40(1), 233-250.

Jiang, Y., \& Wagner, L. C. (2004). What is learned in spatial contextual cuing-Configuration or individual locations? Perception \& Psychophysics, 66(3), 454-463.

Jiang, Y. V., Won, B. Y., Swallow, K. M., \& Mussack, D. M. (2014). Spatial reference frame of attention in a large outdoor environment. Journal of Experimental Psychology Human Perception and Performance, 40(4), 1346-1357.

Karn, K. S., Møller, P., \& Hayhoe, M. M. (1997). Reference frames in saccadic targeting. Experimental Brain Research, 115(2), 267-282.

Kerns, J. G., Cohen, J. D., MacDonald, A. W., Cho, R. Y., Stenger, V. A., \& Carter, C. S. (2004). Anterior cingulate conflict monitoring and adjustments in control. Science, 303(5660), 1023-1026.
Knapen, T., Rolfs, M., Wexler, M., \& Cavanagh, P. (2010). The reference frame of the tilt aftereffect. Journal of Vision, 10(1), 8.

Knapen, T., Rolfs, M., \& Cavanagh, P. (2009). The reference frame of the motion aftereffect is retinotopic. Journal of Vision, 9(5), 16.

Kristjánsson, Á., \& Campana, G. (2010). Where perception meets memory: a review of repetition priming in visual search tasks. Attention, Perception, \& Psychophysics, 72(1), 5-18.

Kristjánsson, Á., Mackeben, M., \& Nakayama, K. (2001). Rapid, objectbased learning in the deployment of transient attention. Perception London, 30(11), 1375-1388.

Kristjánsson, Á., \& Nakayama, K. (2003). A primitive memory system for the deployment of transient attention. Perception \& Psychophysics, 65(5), 711-724.

Kumada, T., \& Humphreys, G. W. (2002). Cross-dimensional interference and cross-trial inhibition. Perception\& Psychophysics, 64(3), 493-503.

Lamy, D., Yashar, A., \& Ruderman, L. (2010). A dual-stage account of inter-trial priming effects. Vision Research, 50(14), 1396-1401.

Leber, A. B., Lechak, J. R., \& Tower-Richardi, S. M. (2013). What do fast response times tell us about attentional control? Journal of Vision, 13(3), 1-12.

Lee, J., \& Shomstein, S. (2013). The differential effects of reward on space-and object-based attentional allocation. The Journal of Neuroscience, 33(26), 10625-10633.

Lin, Z. (2013). Object-centered representations support flexible exogenous visual attention across translation and reflection. Cognition, 129(2), 221-231.

Lin, I. F., \& Gorea, A. (2011). Location and identity memory of saccade targets. Vision Research, 51(3), 323-332.

Lin, Z., \& He, S. (2012). Automatic frame-centered object representation and integration revealed by iconic memory, visual priming, and backward masking. Journal of Vision, 12(11), 24.

Logan, G. D. (1988). Toward an instance theory of automatization. Psychological Review, 95(4), 492.

Maljkovic, V., \& Nakayama, K. (1994). Priming of pop-out: I. Role of features. Memory \& Cognition, 22(6), 657-672.

Maljkovic, V., \& Nakayama, K. (1996). Priming of pop-out: II. The role of position. Perception \& Psychophysics, 58(7), 977-991.

Maljkovic, V., \& Nakayama, K. (2000). Priming of popout: III. A shortterm implicit memory system beneficial for rapid target selection. Visual Cognition, 7(5), 571-595.

Marr, D., \& Nishihara, H. K. (1978). Representation and recognition of the spatial organization of three-dimensional shapes. Proceedings of the Royal Society of London B: Biological Sciences, 200(1140), 269-294.

Mathôt, S., \& Theeuwes, J. (2010). Gradual remapping results in early retinotopic and late spatiotopic inhibition of return. Psychological Science, 21(12), 1793-1798.

McRae, K., Butler, B. E., \& Popiel, S. J. (1987). Spatiotopic and retinotopic components of iconic memory. Psychological Research, 49(4), 221-227.

Melcher, D., \& Colby, C. L. (2008). Trans-saccadic perception. Trends in Cognitive Sciences, 12(12), 466-473.

Melcher, D., \& Morrone, M. C. (2003). Spatiotopic temporal integration of visual motion across saccadic eye movements. Nature Neuroscience, 6(8), 877-881.

Miller, J. (1988). Components of the location probability effect in visual search tasks. Journal of Experimental Psychology: Human Perception \& Performance, 14(3), 453-471.

Muraven, M., \& Baumeister, R. F. (2000). Self-regulation and depletion of limited resources: Does self-control resemble a muscle? Psychological Bulletin, 126(2), 247.

Pelli, D. G. (1997). The VideoToolbox software for visual pyschophysics: Transforming numbers into movies. Spatial Vision, 10, 437-442. 
Pertzov, Y., Zohary, E., \& Avidan, G. (2010). Rapid formation of spatiotopic representations as revealed by inhibition of return. Journal of Neuroscience, 30(26), 8882-8887.

Rabbitt, P. M., Cumming, G., \& Vyas, S. (1977). An analysis of visual search: entropy and sequential effects. Attention and Performance VI, 363-386.

Satel, J., Wang, Z., Hilchey, M. D., \& Klein, R. M. (2012). Examining the dissociation of retinotopic and spatiotopic inhibition of return with event-related potentials. Neuroscience Letters, 524(1), 40-44.

Schall, J. D. (1995). Neural basis of saccade target selection. Reviews Neuroscience, 6, 63-85.

Serences, J. T., \& Yantis, S. (2006). Selective visual attention and perceptual coherence. Trends in Cognitive Sciences, 10(1), 38-45.

Shomstein, S., \& Johnson, J. (2013). Shaping attention with reward effects of reward on space-and object-based selection. Psychological Science, 24(12), 2369-2378.

Smith, A. D., Hood, B. M., \& Gilchrist, I. D. (2008). Visual search and foraging compared in a large-scale search task. Cognitive Processing, 9(2), 121-126.

Smith, A. D., Hood, B. M., \& Gilchrist, I. D. (2010). Probabilistic cuing in large-scale environmental search. Journal of Experimental Psychology: Learning, Memory, and Cognition, 36(3), 605.

Tipper, S. P., \& Behrmann, M. (1996). Object-centered not scene-based visual neglect. Journal of Experimental Psychology: Human Perception and Performance, 22(5), 1261.
Tipper, S. P., Jordan, H., \& Weaver, B. (1999). Scene-based and objectcentered inhibition of return: Evidence for dual orienting mechanisms. Perception \& Psychophysics, 61(1), 50-60.

Tipper, S. P., Weaver, B., Jerreat, L. M., \& Burak, A. L. (1994). Objectbased and environment-based inhibition of return of visual attention. Journal of Experimental Psychology: Human Perception and Performance, 20(3), 478-499.

Umiltà, C., Castiello, U., Fontana, M., \& Vestri, A. (1995). Objectcentred orienting of attention. Visual Cognition, 2(2-3), 165-181.

Vaziri Pashkam, M., \& Cavanagh, P. (2009). Spatiotopic priming in visual search. Journal of Vision, 9(8), 1206.

Walthew, C., \& Gilchrist, I. D. (2006). Target location probability effects in visual search: An effect of sequential dependencies. Journal of Experimental Psychology: Human Perception and Performance, $32(5), 1294$

Wolfe, J. M. (1994). Guided search 2.0 a revised model of visual search. Psychonomic Bulletin \& Review, 1(2), 202-238.

Yashar, A., \& Lamy, D. (2010). Intertrial repetition affects perception: The role of focused attention. Journal of Vision, 10(14), 3.

Zhang, E., \& Li, W. (2010). Perceptual learning beyond retinotopic reference frame. Proceedings of the National Academy of Sciences of the United States of America, 107(36), 15969-15974.

Zhang, E., Zhang, G. L., \& Li, W. (2013). Spatiotopic perceptual learning mediated by retinotopic processing and attentional remapping. European Journal of Neuroscience, 38(12), 3758-3767. 